\title{
EI enfoque totalizador en los educadores del siglo XXI
}

\author{
PhD. Aleida Márquez Rodríguez \\ Universidad de Oriente \\ alejandroc@uo.edu.cu
}

\begin{abstract}
Palabras claves: actividad pedagógica profesional, nuevo estilo de Recibido: 3 de marzo de 2017 pensamiento, transdisciplinariedad, enfoque totalizador.
\end{abstract}

Keywords: professional pedagogical activity, new style of thinking,

Aceptado: 10 de abril de 2017 transdisciplinary, totalizing approach

\section{RESUMEN}

Las aceleradas y crecientes complejidades en las que estamos inmersos, en la era de las sociedades del conocimiento y de la información, en los órdenes científico-técnico, político, económico, social, ambiental, cultural, le plantean al profesional de la educación la necesidad de elevar su preparación pedagógica, psicológica, sociológica, ideológica, ética, entre otras, para atender la diversidad de problemáticas e imprevistas contingencias que emergen en su significativa labor cotidiana. En el trabajo se presenta una sistemati- zación como resultado de investigaciones realizadas y aplicadas durante más de cuatro décadas, se muestra el tránsito de un enfoque analítico hacia un enfoque totalizador en las concepciones epistemológicas. Asimismo, se fundamenta un nuevo estilo de pensamiento holístico complejo, interdisciplinar y transdisciplinar cuyos aportes teóricos y metodológicos contribuyen a actualizar y elevar la eficiencia de la actividad pedagógica profesional de los educadores, acordes con las actuales y futuras exigencias profesionales y sociales de este nuevo siglo.

\begin{abstract}
The accelerated and growing complexities in which we are immersed in the era of knowledge and information societies, in scientific, technical, political, economic, social, environmental and cultural orders demand of professional educators to elevate its pedagogical, psychological, sociological, ideological and ethical preparation, among others, to attend to the diversity of problematic and unforeseen contingencies that arise in its important daily work. The work presents a systematization as a result of a research carried out and applied for more than four decades, shows the transition from an analytical approach to a totalizing approach in epistemological conceptions, is based on a new holistic, complex, interdisciplinary and transdisciplinary thinking style whose contributions theoretical and methodological aspects contribute to updating and increasing the efficiency of the professional pedagogical activity of educators, in accordance with the current and future professional and social demands of this new century.
\end{abstract}




\section{INTRODUCCIÓN}

"Las tendencias actuales de la educación superior y de la sociedad del conocimiento están inmersas en un escenario de cambios radicales del sistema-mundo (...). El desarrollo de la sociedad del conocimiento tiene lugar en un marco signado por la complejidad y la incertidumbre, como nuevo paradigma científico y teórico, en una transición sistémica desde el punto de vista histórico." (López ,2009).

Las grandes metas del siglo XX, planteadas en importantes cónclaves en relación a las necesidades educacionales en el Tercer Mundo, en la mayoría de los países no se cumplieron, pero en Cuba, a pesar de las limitaciones económicas que afrontamos, existe una voluntad política orientada a la preparación de sus habitantes para convivir en un ambiente de cultura integral, justicia social, paz y desarrollo sostenible.

A partir del triunfo de la Revolución, en el año 1959, se han alcanzado logros significativos en las ciencias en general y, en particular, en la esfera educacional. En este lapso, como evidencias del sostenido interés de perfeccionamiento, se han generado tres revoluciones educacionales; pues de una población, que en la década de los 60 alcanzaba casi un 10\% de analfabetismo, hoy exhibe al mundo una población de la cual más del $70 \%$ ha culminado el nivel medio y el 33\% ha alcanzado alguna especialización como obrero calificado, técnico medio o graduado universitario según lo declarado en el 2013 por la Oficina Nacional de Estadísticas. Se han creado instituciones científicas que muestran un alto prestigio por resultados obtenidos y aplicados con éxito a nivel nacional y más allá de nuestras fronteras.

No obstante, en la esfera educacional se reconocen insatisfacciones en el despliegue de la actividad pedagógica profesional; pues han subsistido corrientes, tendencias, teorías y modelos educativos tradicionales que la han sustentado, imprecisiones en el dominio de su estructura, falta de consenso en el proceder metodológico, en particular, en el tratamiento de las habilidades, que han limitado su desarrollo y han requerido ser actualizadas para que se correspondan con las dinámicas y cambiantes condiciones que prevalecen en este nuevo siglo. Es preciso formar docentes con ánimo y competencias nuevas para encarar los desafíos que enfrenta la educación del siglo XXI en el contexto actual de los cambios políticos, sociales, económicos, culturales, tecnológicos, del mercado laboral y de la sociedad del conocimiento y la información.

\section{DESARROLLO}

Se valoraron las aceleradas y crecientes complejidades que afloraban a las puertas de la sociedad del conocimiento, en el orden científico-técnico y la necesidad de apropiarse, actualizarse y aplicar con celeridad la información; se reflexionó en las insuficiencias en el orden educativo, la multiplicidad de aspectos en que debe estar preparado un profesor para potenciar la formación político-ideológica, la ética profesional, atención a la diversidad, a la inclusión, cultura de paz, educación para la sustentabilidad, prevención de la violencia, prevención de problemas de salud, preparación y actualización en las tecnologías de la información y las comunicaciones (Tics), por solo citar algunos aspectos a atender desde la actividad pedagógica profesional.

En la labor cotidiana de los profesores existieron, además, imprevistas contingencias, diversidad de problemáticas que también debieron atender, no en forma atomizada, sino, desde una visión previsora-preventiva, integradora, oportuna, racional, siguiendo una adecuada lógica didáctico-pedagógica que facilitara alcanzar la necesaria formación integral de los futuros educadores, así como la propia superación en su rango de profesional, insuficientemente logradas en algunos aspectos de su configuración.

Esto permitió constatar que persistían vacíos teóricos y metodológicos, que debían ser precisados; una concepción epistemológica desfasada, que debía ser actualizada para fundamentar y caracterizar el enfoque de una actividad pedagógica en correspondencia con las exigencias imperantes, para atender aspectos esenciales en su configuración, desde una mirada interdisciplinar y transdisciplinar, capaz de dar efectivo tratamiento a las complejidades y múltiples contingencias que se debían enfrentar por los profesionales de la educación. 
Se percibió la existencia de una invisibilidad y negación epistemológica en el reflejo de la realidad educativa en el pensamiento de los educadores, que impedía o limitaba, desde una mirada acentuadamente empirista, el tratamiento necesario a dicha realidad, para dar respuestas viables y efectivas que satisficieran las exigencias de la formación profesional en las nuevas condiciones del siglo XXI.

A partir de sistematizar insuficiencias planteadas en diagnósticos realizados en tesis de doctorados y maestrías desde el 2002 hasta el 2012, de diferentes temáticas e informes de controles a clases, se constató que se reiteraban dificultades tales como:

- Impartición de conocimientos acabados

- $\quad$ Ausencia o insuficiente problematización

- $\quad$ Recurrencia en el tratamiento didáctico focalizado en la dimensión cognoscitiva, en detrimento de otras dimensiones para la formación integral personal y profesional.

- Insuficiente estimulación de la motivación en las clases

- Desaprovechamiento de contenidos y actividades para desarrollar y consolidar valores.

- Tratamiento descontextualizado de contenidos, sin vínculos con la actualidad local, nacional e internacional.

- Desaprovechamiento de potencialidades intradisciplinarias e interdisciplinarias de los contenidos

- Enfoques parcializados o fraccionados para enfrentar o solucionar situaciones conflictivas y complejas, entre otras.

La lógica dialéctica marxista demuestra que tanto por su contenido como por su forma o estructura, el pensamiento está sujeto al desarrollo histórico. Los niveles de complejidad creciente de la práctica social influyen de manera determinante en el proceso de formación de la estructura lógica del pensamiento, dando lugar a la aparición de diferentes estilos de pensamiento en correspondencia con cada nueva época histórica. (Rodríguez , 2001).

Desde nuestra experiencia, se puede considerar que el pensamiento se ha mantenido permeado de representaciones de modelos obsoletos, aplicados durante muchos años con resultados que han alcanzado soluciones más o menos satisfactorias para situaciones históricas concretas en determinados momentos, pero que en la actualidad, cada vez más, limitan una actuación en correspondencia con las crecientes y objetivas complejidades de los procesos educativos, inmersos en un contexto plurideterminado. Se hace necesario incidir en esta dificultad, de la cual parece no estar suficientemente consciente la mayoría de los profesionales de la educación.

Se realizaron reflexiones sobre planteamientos avalados por especialistas convocados por la UNESCO para establecer un debate internacional sobre las ideas de Edgar Morín en el contexto de su visión sobre "Pensamiento Complejo" (1999), con el fin de reorientar la educación hacia un futuro viable para un desarrollo sostenible.

La autora se identificó con criterios planteados por Celia Romero en "Paradigma de la complejidad, modelos científicos y conocimiento educativo" (2000) cuando expresa:

El comportamiento caótico de un sistema se visualiza cuando ante dos condiciones iniciales similares, un sistema evoluciona de manera radical o parcialmente diferente a como lo hacen otros sistemas que parten de las mismas condiciones. Como puede inferirse los fenómenos de la educación y del aprendizaje pueden categorizarse como fenómenos caóticos. 
También, se consideraron importantes las ideas expresadas por Antoni Colom (2002):

La teoría del caos cumple la misión de mostrarnos tal cual es la realidad de la educación: fenómenos irreversibles en lo temporal de alta complejidad, en lo absoluto lineal, con diferencias significativas en su punto de partida (la diversidad genética y social, biológica y psicológica, cultural y de clase que se da entre niños de las escuelas infantiles), impredecible, de alta contingencia, continuamente estructurante y por estructurar, dinámica y en definitiva caótica.

Las características subrayadas son generales y no privativas de unos u otros sujetos. Esto permitió reelaborar y enriquecer determinadas ideas percibidas y, en forma incompleta, trabajadas en investigaciones precedentes, en la dinámica de diferentes procesos o en las interacciones de los sujetos, sin tener entonces suficientes fundamentos.La evolución de las reflexiones realizadas permitió un salto hacia una posición más esencial, lo que significó ascender a relaciones de un nivel superior en su fundamentación.

Se consideró necesario concebir una actividad pedagógica no solo interdisciplinar, también transdisciplinar, teniendo en cuenta de manera integrada e interdependientes aspectos biológicos, psicológicos, sociológicos, éticos, ambientales, salubristas, culturales, históricos, ecológicos, de prevención, entre otros, y saberes empíricos sistematizados, es decir, con un firme intento de transformar la racionalidad reduccionista, altamente inclinada al unilateralismo, al fraccionamiento o al mecanicismo, hacia una racionalidad flexible, multidimensionada, totalizadora, expresada en procesos que reflejen recurrentes uniones, bifurcaciones, contradicciones y transformaciones.

La interdisciplinariedad la comprendemos como un proceso de interpenetración cognosci- tiva integradora de dos o más disciplinas (síntesis integradora disciplinaria) que comparten teorías y problemas de investigación, métodos y técnicas de procesamiento de conocimien- tos, principios y leyes generales, redes conceptuales y realizaciones tecnológicas particula- res.

Implica la fusión en una nueva expresión conceptual estructural, explicativa y temática, pero con sentido unitivo de cuerpo de ideas y conocimientos con nuevos significados, elaborando nuevos constructos y nuevos conceptos (unicidad epistémica conceptual y sistémica). Es una forma compleja de construcción de conocimientos ya listos o validados, estableciéndose sistematizaciones descriptivas y explicativas de una nueva concepción del asunto y versiones teóricas de un nuevo tipo.

Los esfuerzos interdisciplinares, aun cuando nos ayudan a prevenir los excesos de especialización y de compartimentalización del saber, no resultan suficientes para dar cuenta de la complejidad de los fenómenos, sean biofísicos o socioculturales. Es decir, la interdisciplinariedad aun no resulta una estrategia efectiva para dar cuenta del entrelazamiento de las múltiples dimensiones sobre las que se organiza la realidad como un todo, o lo que es lo mismo, como una unidad interrelacionada (complejidad).

La transdisciplinariedad, que exige como complemento la interdisciplinariedad, es un proceso de organización, estructuración, construcción y desarrollo de conocimientos, fundado en la elaboración de sistemas temáticos de descripciones y explicaciones con conocimientos provenientes, tanto de disciplinas diversas como de sectores del hacer y del saber (por ejemplo de literatura, artísticos) no organizados en forma de disciplinas

A partir del análisis de sistemas de principios utilizados tradicionalmente de Klinberg (1972), Labarrere y Valdivia (1988) y Addine, (2002) se valoró que se requería incorporar nuevas elaboraciones que pudieran guiar una actuación con una nueva racionalidad.

Se valoraron postulados que se asumieron y contextualizaron como guías en la nueva construcción teórica: 
El enfoque totalizador en los educadores del siglo XXI

- $\quad$ El principio de la existencia de procesos de naturalezas diferentes integrados en un sistema o todo organizado y en funcionamiento.

- $\quad$ El principio de las relaciones dinámicas entre lo aleatorio (lo indeterminado, lo casual) y la necesidad (lo predeterminado, lo probabilístico)

- La transdisciplinariedad, que exige la complementariedad de estudios interdisciplinarios.

La integración y adecuación de estas ideas rectoras, sirvieron para darle tratamiento a la diversidad de procesos educativos complejos que se enfrentan en la realidad educativa.

Se ha constatado que no es suficiente plasmar en documentos normativos, diseñar, orientar, incluso aplicar diversas alternativas metodológicas; se hacen necesarios cambios raigales, que conduzcan a la apropiación de nuevas posiciones epistemológicas, de una nueva estructuración lógica, que permita la transformación de las mentalidades hacia un estilo de pensamiento acorde con las actuales complejidades de los procesos, que se proyecte en el despliegue de la actividad pedagógica profesional y permita dirigir la formación de la personalidad competente e integral con las características que necesitamos, a las puertas de la era del conocimiento:

(...) se refiere a cómo nos acercamos al conocimiento de la realidad y cómo esta es percibida y comporta con una intencionalidad totalizadora en cuanto a elementos que la componen

(...), su objetivo es hablarnos de cómo son las cosas y los acontecimientos en la realidad: globales y a su vez unitarios, complejos y compuestos por múltiples elementos sumamente interrelacionados. (Zabala, 1989).

La concepción epistemológica asumida, en sus fundamentos posee componentes del paradigma de la complejidad de Morín (1999), de la teoría histórico cultural de Vigotsky (1996) potenciada con la teoría holístico configuracional de Fuentes (2002) que contribuyó a transitar de un reflejo de la realidad con una comprensión empírica, analítica, basada en una lógica lineal, hacia un pensamiento que tuvo en cuenta interacciones y conexiones entre habilidades intelectuales de diferentes grados de agudeza y profundidad con habilidades lógico-dialécticas capaces de captar con mayor precisión la dinámica contradictoria y transformadora de diferentes procesos.

Se asumió también como presupuesto el carácter consciente, holístico- dialéctico y complejo a partir de que los sujetos interpreten la realidad como una totalidad, en sus multivariadas contradicciones, dialogar con la riqueza y diversidad de esta con un enfoque interdisciplinar y transdisciplinar.

Asimismo se corroboró la necesidad de propiciar un nuevo estilo de pensamiento científico, basado en la aprehensión, mediante habilidades lógicas dialécticas (que incluyen las lógicas formales), en el profesional de la educación, que es el principal director y orientador de los procesos formativos en las instituciones educativas.

El concepto estilo de pensamiento científico, desde una perspectiva general, fue introducido por el físico alemán Max Born (1882-1970) en su obra La Física en la vida de mi generación, quien entendía que este se manifestaba en las tendencias generales del pensamiento, que se modifican muy lentamente, configuran determinados marcos filosóficos, con ideas características en todas las ramas de la actividad humana, incluyendo las ciencias. Desde una perspectiva personal, es considerado como el modo específico que tiene el sujeto de utilizar sus capacidades cognitivas.

Es necesario que los docentes se apropien de un estilo de pensamiento, que facilite comprender e interpretar dialécticamente procesos complejos, multicondicionados y plurideterminados que se manifiestan en redes de relaciones, incluso no de la misma naturaleza, aplicarlo en la solución de problemáticas educativas y no estancarse en un reduccionismo metodológico. 
En una comprensión holística dialéctica y compleja de la totalidad, se requiere captar los procesos en su dinámica, en sucesivas evoluciones, bifurcaciones, en continuas aproximaciones cognoscitivas de reflexión, comprensión, explicación e interpretación, a partir de sus contradicciones, para su acertada transformación; pero no se debe identificar lo complejo solo al considerar la existencia de numerosos factores para la solución de un problema. La complejidad, en la comprensión holística dialéctica, consiste en asumir que en la dinámica de los objetos, entendidos como procesos, "se generan en su movimiento relaciones deterministas cualitativamente diversas, no reducibles, en principio, a las relaciones deterministas causales lineales y cuantitativas. La complejidad es, ante todo, diversidad cualitativa de relaciones deterministas." Chibás (2009).

En la concepción que propone la autora lo holístico supone totalidades dinámicas, integradas por procesos configurados como unidades interactivas e interconectadas; lo complejo destaca interrelaciones de diferentes naturalezas, portadoras de propiedades emergentes que no se deducen de sus componentes; lo dialéctico supone la sucesiva emergencia, resolución o transformación de contradicciones.

Estos presupuestos establecen bases epistemológicas de mayor esencialidad, que sustentan y posibilitan el despliegue de la actividad pedagógica profesional con un enfoque totalizador.

Se entiende por enfoque totalizador:

El marco teórico-metodológico de referencia que permite desplegar un estilo de pensamiento científico (lógico-dialéctico), resultado de la interacción de procesos holísticos y complejos de la realidad natural, social y procesos subjetivos, concebidos como totalidades dinámicas, multidimensionadas y plurideterminadas, portadoras de propiedades emergentes que no se deducen de sus elementos componentes, con la finalidad de analizar, reflexionar, comprender, explicar, interpretar y transformar fenómenos de diferentes naturalezas. (Márquez, 2015).

Enmarcar el despliegue de la actividad pedagógica en este enfoque requiere de condiciones previas tales como: disposición para asumir actitudes y acciones críticas, reflexivas y metacognitivas en el trabajo de diseño y ejecución de los contenidos disciplinares para reconocer si se avanza, tanto en los propósitos transformadores ideales como conductuales, en la práctica; concebir el proceso de enseñanza aprendizaje con carácter interdisciplinar y transdisciplinar; es recomendable promover que los cambios sean resultado del trabajo científico metodológico, lo cual asegurará mayor confiabilidad, aunque no estará exento de modificaciones o correcciones sistemáticas.

\section{El proceder metodológico se puede apoyar en procedimientos tales como:}

- $\quad$ Entrenamiento para potenciar el desarrollo de habilidades lógico-dialécticas (la captación, el reflejo, la representación y análisis no lineal de la realidad educativa), con una perspectiva holística y compleja.

A partir de identificar, demostrar y ejemplificar:

- $\quad$ Situaciones o procesos educativos con posibles repercusiones plurideterminadas.

- $\quad$ Situaciones curriculares que requieren de contenidos de varias disciplinas, de diferentes perspectivas epistemológicas y saberes no disciplinares.

- $\quad$ Situaciones comunicativas que requieran una visión holística para su tratamiento o solución, entre otras que pudieran seleccionarse.

- La contextualización epistemológica del trabajo disciplinar teniendo como guías para las transformaciones, los valores epistémicos del objeto de la profesión y el modelo del profesional, así como las relaciones entre las diferentes disciplinas para alcanzar representaciones interdisciplinares, dialécticas y complejas de los conocimientos. 
- La contextualización epistemológica como actividad, implica una nueva orientación del trabajo didáctico de las diferentes disciplinas para su integración. La integración es de tal naturaleza, que los contenidos de una disciplina implican relaciones de significación en los contenidos de otra y viceversa. Establecer las relaciones entre disciplinas diversas como "orden de composición y unión" para conformar los contenidos requiere, además, consolidar los vínculos reales de comunicación que se establecen entre sus docentes: entre los colectivos de carrera y de años, así como los intercambios periódicos que sostengan los docentes entre si.

- $\quad$ El desarrollo didáctico complejo que persigue una nueva visión didáctica, la cual exige una comunicación interactiva, interdisciplinar entre los docentes de las ciencias natu- rales, sociales, la filosofía y del ejercicio de la profesión para la selección de conocimientos y metodologías colegiadas. Por ello resulta en extremo importante, la combinación didáctica de los problemas profesionales, desde el tratamiento teórico comprensivo de los problemas prácticos y la traducción práctica de los problemas que incitan y sugieren la comprensión hermenéutica.

Un ejemplo que puede ilustrar esta situación lo ofrece la estructuración de la habilidad de modelar que puede desarrollarse como sucesivos momentos comprensivos:

a)como representación de las propiedades empíricas del objeto;

b) como modelo representativo de las relaciones sistémicas del objeto;

c) como modelo explicativo del comportamiento tendencial del objeto;

d) como modelo interpretativo dialéctico hermenéutico de las cualidades emergentes del objeto como proceso a partir de la consideración de sus contradicciones internas.

De igual modo, la habilidad de interpretar puede diseñarse a partir de diferentes niveles jerárquicos.

- Universalización práctica profesional a partir de elaboración y solución de tareas, problemas profesionales, despliegue de proyectos interdisciplinares y transdisciplinares (saberes no incluidos en las disciplinas), donde las exigencias se combinen para desarrollar habilidades lógico-dialécticas y estrategias totalizadoras.

Las técnicas constitutivas fundamentales son las siguientes:

- $\quad$ Orientación didáctica hacia los problemas profesionales que tienen connotación universal

- $\quad$ Socialización de resultados

- $\quad$ Elaboración de proyectos de práctica profesional deavanzada

El hecho de promover un enfoque totalizador no excluye que en la dirección del proceso pedagógico se tengan en cuenta las funciones establecidas para ello, (docente-metodológica, de superación e investigación y de orientación), pues debe lograrse una necesaria complementación en el despliegue de la actividad pedagógica profesional.

El primer procedimiento debe ser objeto de atención priorizada; los demás deben interactuar, no en el sentido analítico tradicional (en un orden lineal, jerárquico o algorítmico) sino dialécticamente, es decir, en cualquiera de las variantes didácticas, en ocasiones todos pueden estar presentes, aunque con diferentes implicaciones.

Es preciso significar que se alcanzaron resultados, que evidenciaron tendencias positivas en el cambio de mentalidad (estilo de pensamiento) ya que su implementación más efectiva requiere transformaciones, no solo a nivel de la actuación profesional de base (colectivos docentes), sino a nivel institucional y otros nivelessuperiores. 
Se participó en eventos locales, provinciales e internacionales, particularmente en los Congresos de Pedagogía Internacional donde se impartieron cursos preevento en los años 2007 y 2009, dando a conocer el nuevo enfoque totalizador, asumido y aplicado desde la perspectiva de la complejidad, como forma de enriquecer el quehacer pedagógico. También se participó, como conferencista, en los eventos estaduales de Pedagogía $(2008,2010)$ y Universidad (2009) en Maracaibo, estado Zulia, Venezuela.

Se impartieron conferencias en pregrado en la Licenciatura Pedagogía Psicología, cursos de postgrado sobre Creatividad, Psicología del Aprendizaje e Investigación Educativa en la maestría de la Universidad de Ciencias Pedagógicas "Frank País García"; en la maestría en educación en la República Bolivariana de Venezuela (2007) y en el programa de formación doctoral conveniado por el IPLAC con la Universidad Bolivariana de Venezuela (2009- 2012), en la Escuela Superior del Ministerio del Interior (Santiago de Cuba), en la Facultad de las Ciencias de la Cultura Física y el Deporte, (ídem), en el Instituto Tecnológico General Milanés (provincia Granma), en la Universidad de Ciencias Pedagógicas. (Ídem).

Se participó como miembro en los Proyectos del Centro de Estudios Juan Bautista Sagarra Blez: "La formación pedagógica de los profesionales de la educación" y "Formando al educador del siglo XXI" para los cuales se elaboraron materiales para la comprensión y despliegue interdisciplinar, así como estrategias para el desarrollo de la metacognición en la actividad pedagógica profesional.

Se dirigió el proyecto para superar a los maestros de la escuela de autismo "William Soler" de Santiago de Cuba. (2006 - 2010) Los mismos fueron capacitados y entrenados con las nuevas elaboraciones teórico-metodológicas para el despliegue de una actividad pedagógica creativa, con enfoque totalizador y asesorados con el objetivo de realizar sus maestrías, las cuales defendieron con éxito.

También, se intercambiaron aspectos de la nueva concepción de la actividad pedagógica profesional en la dependencia provincial del Centro Coordinador para la Formación y el Desarrollo del Capital Humano del Ministerio de la Informática y las Comunicaciones, en la filial de la provincia Santiago de Cuba, con muy buena aceptación.

Como resultado de la socialización de este enfoque totalizador, afloraron transformaciones en el despliegue de la actividad en la formación pedagógica profesional reflejados, fundamentalmente, en los aprendizajes de los estudiantes de formación inicial, así como en maestros de educación primaria y de educación especial, que dan cuenta de las efectivas posibilidades de su aplicación sistemática, para gradualmente alcanzar resultados más integrales.

A través de las sistemáticas reflexiones, experiencias investigativas y a partir de indicado- res intencionalmente seleccionados fue evidente que se produjeron saltos cualitativos en las elaboraciones teórico-metodológicas de la actividad pedagógica profesional, las cuales permitieron a la autora precisar que su concepción transitó de un enfoque analítico a un enfoque totalizador, no obstante, aún se requerirán nuevas reflexiones y enriquecimientos.

La actividad pedagógica profesional con enfoque totalizador se define como:

Aquella concebida desde una perspectiva humanista, holístico-dialéctica, interdisciplinar y transdisciplinar, en una relación entre lo ético y lo científico, que permite penetrar en la lógica de los procesos formativos, para estimular potencialidades que favorezcan el desarrollo de sujetos reflexivos, cultos, integrales, acordes con la complejidades de su tiempo y su contexto. (Márquez, 2016).

Su carácter facilitador de aprendizajes y autoaprendizajes, se orienta al cumplimiento, al menos, de las funciones docente metodológica, de orientación e investigación y superación, trasciende el cumplimiento de las funciones antes referidas, (se propone propuso incluir la función previsora-preventiva) por su condición de transformar un objeto multidimensionado y plurideterminado (la realidad educativa), para enfrentar y resolver múltiples complejidades, que se concretan en una red transdisciplinar de competencias profesionales, teniendo en cuenta una diversidad de factores objetivos, subjetivos y contextuales que son necesarios 
atender en su dinámica interactiva e interconectada, para alcanzar un desempeño de excelencia educativa.

Entre las múltiples dimensiones en que debe proyectarse, están los siguientes:

- La dirección del proceso pedagógico para la formación profesional se sustenta en un estilo de pensamiento científico (lógico-dialéctico) holístico y complejo, con una visión interdisciplinar, transdisciplinar, contextualizada y creativa.

- $\quad$ Autoperfeccionamiento profesional, a partir de la metacognición, la pronosticación, valoración crítica, reflexiva y autorregulada de su actividad.

- $\quad$ Comunicación e interrelaciones que propicien un clima favorable en el proceso formativo.

- Estimulación didáctico-pedagógica de procesos y resultados cognitivos, afectivos, volitivos, éticos, valorativos en su integración dialéctica.

- Instrumentación del protagonismo para aprender a aprender, incluidas las posibilidades de dominio tecnológico.

- $\quad$ Atención sistemática y diferenciada a la diversidad y a lainclusión.

- Incorporación de la función previsora-preventiva y su despliegue en diferentes áreas, según necesidades individuales-colectivas y contextuales.

- $\quad$ Transversalización de la educación para la sustentabilidad, para la paz.

- $\quad$ Apropiación y aporte a la socialización de experiencias.

La instrumentación de este nuevo enfoque de la actividad pedagógica profesional es una alternativa que tiene en cuenta las actuales exigencias en la preparación de los profesionales, pero no excluye la consideración de otras alternativas. Requiere de una preparación gradual y sistemática, a través de diferentes vías como son: conferencias, seminarios, talleres, cursos de postgrado, fundamentalmente actividades colegiadas desde el sistema de trabajo metodológico interdisciplinar y transdisciplinar en los diferentes niveles, autopreparación con fines de autoperfeccionamiento, entre otros.

\section{CONCLUSIONES}

Las sistemáticas reflexiones y los resultados alcanzados en la solución de una estimable diversidad de problemáticas educativas evidenciaron que el proceso pedagógico debe tener en cuenta, en armoniosa interconexión, los requerimientos de la era del conocimiento y la relación que se establece con la dinámica actual de factores socioeconómicos, políticos, ideológicos, científicos, técnicos, teóricos, prácticos, éticos y culturales en general, para lograr un proceso formativo integral en los profesionales de la educación, que pueda calificarse como pertinente, y facilite respuestas viables a las necesidades actuales y perspectivas de la sociedad.

Se constataron recurrentes deficiencias en el desempeño de los profesionales de la educación, con diferencias espacio-temporales, en variadas dimensiones de la actividad pedagógica, que justificaron la necesidad de realizar cambios en el orden epistemológico, con el objetivo de generar transformaciones en el pensamiento y su concreción instrumental, acordes con las actuales complejidades sociales.

Los sistemas de principios, las conceptualizaciones, caracterizaciones e instrumentaciones aportadas han contribuido a una mejor comprensión de la actividad pedagógica profesional; han enriquecido desde la teoría, fundamentalmente, contenidos pedagógicos, psicológicos y didácticos, así como han incrementado la motivación, apropiación y aplicación de conocimientos en los profesionales de la educación, tanto en su formación inicial como en el proceso formativo en general. 


\section{REFERENCIAS BIBLIOGRÁFICAS}

Addine, F. (2002). Principios para la dirección del proceso pedagógico. En Compendio de Pedagogía. La Habana, Editorial Pueblo y Educación.

Cabrera. M. (2004). Recensión. "Conversación”. Revista Interdisciplinaria de reflexión y ex-periencia.

Chibás, J. D. (2009). El desarrollo de habilidades lógicas como estilo de pensamiento en los estudiantes de la carrera Licenciatura en Cultura Física de la Facultad de Santiago de Cuba. Tesis en opción al título de doctor en ciencias pedagógicas. Centro de Es- tudios M. F. Gran, Universidad de Oriente, Santiago de Cuba, Cuba, p. 20.

Colom, A. (2002). La (de)construcción del conocimiento pedagógico. Nuevas perspectivas en teoría de la educación. Ediciones Paidos, Barcelona, Buenos Aires, México, pp. 159-160.

Fuentes, H. (2002). La teoría holística configuracional y su aplicación a la Didáctica de la Edu- cación Superior. Centro de Estudios de la Educación Superior M. F. Gran: Universidad de Oriente, en soporte digital.

Klingberg. L. (1972). Introducción a la Didáctica General. La Habana: Editorial Pueblo y Edu- cación.

Labarrere, G. y G. Valdivia, (1988). Pedagogía. La Habana: Editorial Pueblo

y Educación. López, F. (2009). Educación superior y sociedad del

conocimiento. Tendencias actuales -

Asesoría Académica Universidad de Naciones Unidas. Revista

Temas, No. 57, pp. 4 -15.

Márquez, A. (2016). La actividad pedagógica profesional: de un enfoque analítico a un enfo- que totalizador. Tesis en opción al título de doctor en ciencias, Universidad de Hol- guín, Cuba, p.128.

Morín, E. (1999). Los siete saberes necesarios para la educación del futuro Recuperado de: http:// www.cubaeduca.rimed.cu.-

Oficina Nacional de Estadísticas e Información. (2012) Informe Final del Censo de Población y Vivienda. Resumen Adelantado. Publicado en Periódico Juventud Rebelde, viernes 8 de noviembre de 2013, pp. 45.

Oficina Regional de la UNESCO. (2002). Proyecto Regional de Educación para América La- tina y el Caribe (PRELAC) y los mecanismos de seguimiento. Aporte al Congreso Internacional Pedagogía 2003. La Habana, Cuba, p.17.

Rodríguez, Z. (2001). Obras. Tomo 2, segunda edición. La Habana: Editorial Pueblo y Edu- cación, p.88.

Romero Pérez, C. (2000). Paradigma de la complejidad, modelos científicos y conocimiento educativo. Universidad de Huelva. Extraído en mayo de 2007. Disponible en: http:// www3.usal.es. 
Zabala, A. (1989). El enfoque globalizador. En Cuadernos de Pedagogía. Madrid, España: Editorial Fontalba, No.168, p. 19.

Vigotsky, L. (1991). Problemas teóricos y metodológicos de la psicología. Obras Escogidas, Tomo I, Centro de Publicaciones del M.E.C, Ciudad Universitaria, Madrid. 